\title{
Systematic endoscopic assessment of bleeding sites in severe epistaxis: the role of the S-point and the superior epistaxis*
}

\author{
Clarice Naya Loures', Thaiana Carneiro de Castro', Gabriela Ricci Lima Luz \\ Matsumoto', Vinícius de Souza Siebert', Laura Schmitt de Lacerda', Maria \\ Victória de Freitas Miranda', Miguel Soares Tepedino², Leonardo Balsalobre 1,3, \\ Aldo Cassol Stamm³, Eduardo Macoto Kosugi' \\ Rhinology 58: 5, 477 - 481, 2020 \\ https://doi.org/10.4193/Rhin 19.466 \\ *Received for publication \\ December 27, 2019 \\ ' Federal University of Sao Paulo (UNIFESP-EPM), Department of Otorhinolaryngology and Head and Neck Surgery, Sao Paulo, SP, \\ Accepted: February 28, 2020 \\ Brazil \\ 2 Botafogo Polyclinic, Department of Otorhinolaryngology, Rio de Janeiro, RJ, Brazil \\ Edmundo Vasconcelos Hospital Complex, Otorhinolaryngology, Sao Paulo, SP, Brazil
}

\begin{abstract}
Background: Systematic endoscopic assessment (SEA) of bleeding sites is critical for topodiagnosis and treatment of severe epistaxis, which is not limited to the posterior region. A bleeding site originating from the ethmoidal vasculature, the S-point, has recently been described. The aim of this study is to ascertain the prevalence of each bleeding site in severe epistaxis using a SEA protocol that includes the S-point.
\end{abstract}

Methodology: Prospective longitudinal study of 51 severe epistaxis patients who underwent 53 SEA under general anesthesia from April 2018 through March 2019. SEA consisted of use of a rigid nasal endoscope; no reduction in blood pressure; no use of topical vasoconstrictor; systematic search of all regions of the nose. Bleeding sites were assigned to either superior or posterior epistaxis.

Results: At least one bleeding site was identified in 37 evaluations (69.8\%). The S-point was the most common bleeding site (28.3\%), followed by the lateral middle turbinate (9.4\%), non-S-point upper septum (7.5\%), nasal roof (7.5\%), and upper lateral wall (7.5\%). Superior epistaxis was identified in the most of cases (27 SEA, 50.9\%), whereas only 14 SEA (26.4\%) identified posterior epistaxis - fewer than the 16 SEA that did not identify any bleeding sites (30.2\%). There were two recurrences (3.8\%).

Conclusions: Systematic endoscopic assessment effectively identified bleeding sites in $69.8 \%$ of severe epistaxis. The S-point was the most common bleeding site identified (28.3\%). Finally, superior epistaxis corresponded to more than half of the identified bleeding sites, demonstrating the importance of examining this region judiciously in patients with severe epistaxis.

Key words: epistaxis, endoscopy, hemorrhage, hemorrhagic disorders, electrocoagulation

\section{Introduction}

Severe epistaxis can be defined as any episode of massive nasal bleeding that requires urgent or immediate treatment with nasal packing and/or surgical intervention. Severe epistaxis can be life-threatening; blood products and fluid resuscitation may be required to hemodynamic stabilization ${ }^{(1-4)}$. Up to $20 \%$ of ENT emergency room visits for spontaneous epistaxis require nasal packing for hemostasis, and $3.5 \%$ of patients ultimately undergo surgical treatment ${ }^{(5)}$.
Endoscopic assessment of the nasal cavity is mandatory for proper topodiagnosis of severe epistaxis, since bleeding sites are usually located in the superior and posterior regions, and anterior rhinoscopy provides very limited visibility of these areas of the nose $\mathrm{e}^{(6)}$. However, even nasal endoscopy may fail to locate the bleeding site in 6 to $24 \%$ of cases of severe epistaxis ${ }^{(7-10)}$. In addition to enabling more accurate topodiagnosis, nasal endoscopy has revolutionized the treatment of severe epistaxis, allowing selective electrocauterization of the bleeding vascular 
pedicle and thus leaving sphenopalatine artery cauterization only for selected cases ${ }^{(10)}$.

Although the sphenopalatine artery has traditionally been considered the main source of bleeding in severe epistaxis, the ethmoidal arteries are increasingly recognized as major sources; so, controversy exists about which is the major arterial system involved. Recently, the Stamm's S-point, a specific vascular pedicle located in the upper region of the nasal septum near the "axilla" projection of the middle turbinate, has been described ${ }^{(1)}$. This vascular pedicle originates from the septal branches of the ethmoidal arteries and appears to constitute an important source of bleeding in severe epistaxis. Hence, it is recommended that the S-point be always sought in these cases $^{(11)}$. However, there are no available data on the prevalence of the S-point as a source of severe epistaxis. Within this context, the present study aimed to ascertain the prevalence of each bleeding site in severe epistaxis using a systematic endoscopic assessment protocol that includes the S-point.

\section{Materials and Methods}

\section{Study population}

The sample included all patients with severe epistaxis who were attended at the Hospital Sao Paulo (HSP/HU UNIFESP) and underwent systematic endoscopic assessment (SEA) under general anesthesia for topodiagnosis of the bleeding site from April 2018 to March 2019.

Severe epistaxis was defined as any episode of epistaxis with one or more of the following characteristics:

- Active or referred episode of potentially life-threatening bleeding, possibly of superior or posterior origin;

- Need for posterior nasal packing to control bleeding;

- Hemoglobin drop $\geq 2 \mathrm{~g} / \mathrm{dL}$ and/or blood transfusion required;

- Recurrent episodes of epistaxis requiring medical attention and/or recurrent nasal packing.

Patients with severe epistaxis due to recent nasal and/or sinus surgery (up to 3 months), facial trauma, hereditary hemorrhagic telangiectasia, nasal polyps, or nasal and/or sinus tumors were excluded. Also, anterior epistaxis (bleeding source identified by anterior rhinoscopy) was excluded.

\section{Study design}

The present study was approved by the local IRB under number 63539916.2000 .5505 .

A prospective longitudinal design was used. All patients with severe epistaxis who were attended at a specialized ENT emergency department between April 2018 and March 2019 were included. After control of active bleeding with anterior and/or posterior nasal packing, all patients underwent SEA under general anesthesia to search for the bleeding site, as a part of the institutional standard protocol for severe epistaxis. Data collected during this evaluation were used for this study. Systematic endoscopic assessment involved the following principles:

- Use of a 0- or 30-degree rigid nasal endoscope;

- No reduction in blood pressure during general anesthesia;

- No use of any topical vasoconstrictor (adrenaline, oxymetazoline, etc.) during evaluation;

- Systematic search of all regions of the nose for the bleeding site.

The decision to avoid any reduction in blood pressure and any application of topical vasoconstrictor aimed to prevent false negatives, as either situation could cause the bleeding site or the vascular pedicle to disappear. "Bleeding site" was defined as any area with active bleeding or any prominent, visible vascular pedicle with evidence of recent bleeding. Diffusely bleeding or oozing mucosal tears and abrasions were not considered bleeding sites, as they could be due to nasal packing.

The systematic search for the bleeding site aimed to evaluate all areas of the nasal cavity, always in the same order, from upper to lower and from anterior to posterior. Initially, the rigid endoscope was directed superiorly to assess the area superior to the axilla of the middle turbinate (upper lateral wall) and the corresponding septum (S-point). The examiner then proceeded superiorly, assessing the nasal roof and the superior portion of the nasal septum near the roof (nasal roof and non-S-point upper septum) until the superior turbinate was reached. The endoscope was then withdrawn and reintroduced to assess the medial surface of the middle turbinate and corresponding septum (medial middle turbinate and lower septum) to the choana. The middle turbinate was then gently medialized with a saline-soaked cotton pledget to allow visualization the middle meatus (lateral middle turbinate and middle meatus' lateral wall) to the tail of the middle turbinate. Once again, the scope was withdrawn and repositioned to evaluate the inferior turbinate and corresponding septum (medial inferior turbinate and lower septum) all the way to the choana. Finally, the inferior turbinate was gently medialized with a saline-soaked cotton pledget to allow visualization the inferior meatus (lateral inferior turbinate and inferior meatus' lateral wall) (Video 1; available online).

Once the bleeding site was identified, endoscopic cauterization was performed using a Valleylab ${ }^{\circledR}$ monopolar suction coagulator. When more than one bleeding site could be identified, all were cauterized. If no bleeding site was identified, endonasal endoscopic electrocauterization of the sphenopalatine artery (ESPA) was performed, with or without electrocauterization of the anterior ethmoidal artery (EAEA) via Lynch incision.

For purposes of vascular territory comparison, bleeding sites in the S-point, non-S-point upper septum, nasal roof, and upper lateral wall were considered "superior epistaxis". Bleeding sites on the lower septum, choana, medial middle turbinate, lateral middle turbinate, middle meatus' lateral wall, medial inferior 


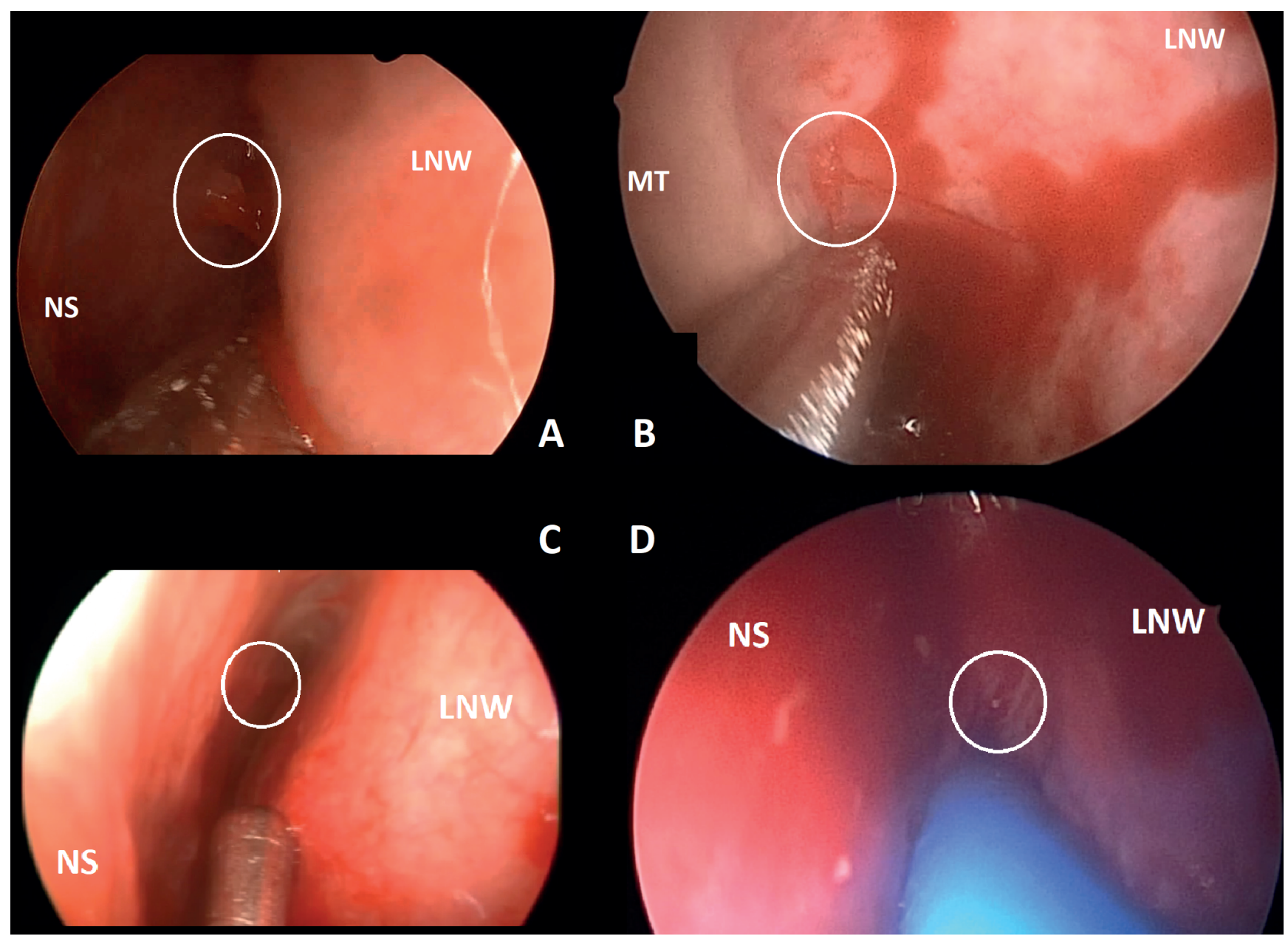

Figure 1. Examples of bleeding sites (in the circles). A: Left nasal cavity, Stamm's S-point. B: Left nasal cavity, lateral middle turbinate. C: Left nasal cavity, non-S-point upper septum. D: Left nasal cavity, nasal roof. NS=nasal septum, LNW=lateral nasal wall.

turbinate, lateral inferior turbinate, and inferior meatus' lateral wall were considered "posterior epistaxis".

\section{Results}

Fifty-eight patients with severe epistaxis were treated and underwent 61 SEA under general anesthesia between April 2018 and March 2019 at a specialized ENT emergency department. Of these, six patients (six SEA, no recurrences) presented with severe epistaxis after nasal and/or sinus surgery and were thus excluded. Another patient (two SEA, one recurrence) had nasal polyps that hindered a complete systematic endoscopic examination and was thus excluded.

The final study sample thus consisted of 51 patients with severe epistaxis who underwent 53 SEA of the bleeding site under general anesthesia. Two of the included patients experienced recurrence of severe epistaxis requiring a second SEA. Thirtytwo patients were male (62.7\%). The mean age was 54.3 years ( $\mathrm{SD}=15.3$ years). The left side was affected predominantly $(n=30,56.6 \%)$. There were no cases of bilateral bleeding. Most patients presented severe anteroposterior bleeding $(n=39$,
73.6\%). Exclusive severe anterior bleeding was seen in 13 patients (24.5\%) and only one patient presented exclusive severe posterior bleeding (1.9\%). Previous medical history included hypertension in 27 patients (50.9\%), aspirin intake in 5 patients (9.4\%), and anticoagulant drugs usage in 3 patients (5.7\%). Most patients were treated with anteroposterior $(n=22,41.5 \%)$ or anterior ( $n=18,34 \%)$ nasal packing. Only 8 patients (15.1\%) were submitted to posterior nasal packing, and 5 patients (9.4\%) did not presented active bleeding during initial consultation and no nasal packing was inserted. Mean initial hemoglobin level was $12.9 \mathrm{~g} / \mathrm{dL}$ (SD=2.8; minimum=6.3 $\mathrm{g} / \mathrm{dL}$ ) and the mean drop of hemoglobin levels was $3.1 \mathrm{~g} / \mathrm{dL}$ ( $\mathrm{SD}=2.4$; maximum=6.9 g/dL). Blood transfusion was required for thirteen patients (24.5\%). The average length of hospital stay was 2.8 days (median=2 days). Most SEA ( $n=37,69.8 \%$ ) effectively identified at least one bleeding site; 33 SEA (62.3\%) identified one site and 4 SEA (7.5\%) identified two sites. In these patients, treatment consisted of electrocauterization of the bleeding site with a Valleylab ${ }^{\oplus}$ monopolar suction coagulator. In one patient (1.9\%), concomitant septoplasty was performed to facilitate access to the bleeding 
Table 1. Description of bleeding sites.

\begin{tabular}{|lll|}
\hline \multicolumn{1}{|c}{ Data } & N & $\%$ \\
\hline Systematic endoscopic assessments & 53 & $100.0 \%$ \\
\hline No bleeding site identified & 16 & $30.2 \%$ \\
\hline Bleeding site identified & 37 & $69.8 \%$ \\
\hline Single site & 33 & $62.3 \%$ \\
\hline Two sites & 4 & $7.5 \%$ \\
\hline Superior epistaxis & 27 & $50.9 \%$ \\
\hline S-point & 15 & $28.3 \%$ \\
\hline Non-S-point upper septum & 4 & $7.5 \%$ \\
\hline Nasal roof & 4 & $7.5 \%$ \\
\hline Upper lateral wall & 4 & $7.5 \%$ \\
\hline Posterior epistaxis & 14 & $26.4 \%$ \\
\hline Lower septum & 0 & $0.0 \%$ \\
\hline Choana & 0 & $0.0 \%$ \\
\hline Medial middle turbinate & 3 & $5.7 \%$ \\
\hline Lateral middle turbinate & 5 & $9.4 \%$ \\
\hline Middle meatus' lateral wall & 3 & $5.7 \%$ \\
\hline Medial inferior turbinate & 2 & $3.8 \%$ \\
\hline Lateral inferior turbinate & 0 & $0.0 \%$ \\
\hline Inferior meatus' lateral wall & 1 & $1.9 \%$ \\
\hline & & \\
\hline
\end{tabular}

site. The S-point was the most commonly identified bleeding site (15 SEA, 28.3\%) (Video 2; available online), followed by the lateral middle turbinate (5 SEA, 9.4\%), non-S-point upper septum (4 SEA, 7.5\%), nasal roof (4 SEA, 7.5\%), and upper lateral wall (4 SEA, 7.5\%) (Table 1 and Figure 1).

Only 16 SEA (30.2\%) failed to identify any bleeding site. These patients underwent ESPA alone (14 procedures, 26.4\%) or ESPA with additional EAEA via Lynch incision (2 procedures, 3.8\%). Regarding the affected territory, superior epistaxis was identified in the most of cases (27 SEA, 50.9\%), whereas only 14 SEA (26.4\%) identified posterior epistaxis - fewer than the 16 SEA that did not identify any bleeding sites (30.2\%) (Table 1). Of the 4 SEA that identified two bleeding sites, 1 (1.9\%) identified two superior epistaxis' sites (nasal roof + non-S-point upper septum), $1(1.9 \%)$ identified two posterior epistaxis' sites (inferior meatus' lateral wall + medial middle turbinate), and 2 (3.8\%) identified concomitant superior and posterior epistaxis' sites (upper lateral wall + medial middle turbinate and middle meatus' lateral wall + non-S-point upper septum).

There were two recurrences of epistaxis (2 SEA, 3.8\%), both in cases in which the initial SEA failed to identify any bleeding site and was followed by ESPA. In the first patient (a 51-year-old male), recurrence occurred after 13 days of initial SEA. The bleeding site was identified in the nasal roof and successfully treated by electrocauterization. In the second patient (a 55-year-old female), relapse occurred on the day following the initial SEA. The S-point was identified as the source of bleeding, and successfully treated by electrocauterization. There were no recurrences when one or more bleeding sites was identified ( $0 \%$ vs. $12.5 \%$; $p=0.09$, Fisher's exact test).

\section{Discussion}

Use of the rigid endoscope has unequivocally revolutionized the topodiagnosis of severe epistaxis ${ }^{(12)}$. When anterior rhinoscopy fails to identify the bleeding site, a more detailed evaluation by nasal endoscopy is recommended, including detailed examination of the lateral nasal wall with angled endoscopes(10). In the present study, systematic endoscopic assessment of the bleeding site under general anesthesia was able to identify the bleeding point in $69.8 \%$ of cases. Other referral centers which recommend an endoscopic nasal inventory have reported high rates of bleeding-site identification, ranging from 76 to $94 \%$ of cases $^{(7,8,10)}$

Kosugi et al. initially described the S-point, an arterial pedicle in the upper septum near the projection of the axilla of the middle turbinate, posterior to the septal body. This landmark corresponded to the bleeding site in nine patients with severe epistaxis examined at three Brazilian hospitals. In all these patients, electrocauterization of the S-point alone led to complete resolution of epistaxis, with no recurrence at 18-month followup $^{(1)}$. A few months later, Turri-Zanoni et al. reviewed 30 cases of severe epistaxis originating from the septal branches of the anterior ethmoidal artery at the level of the axilla of the middle turbinate - the exact same location as the S-point described by Kosugi et al. - at three tertiary referral centers in Italy ${ }^{(11)}$. Both publications were based on lectures by Dr. Aldo Stamm about the importance of the anterior ethmoidal artery as a source of severe epistaxis. However, despite the perceived importance of the S-point in the pathogenesis of severe epistaxis, there are no published data on its prevalence as a bleeding site. The present study is the first to demonstrate a high rate of diagnosis of the S-point as the site of bleeding (28.3\%) when SEA under general anesthesia is performed for severe epistaxis. On the other hand, considering bleeding sites corresponding to the S-point, Chiu and McGarry reported that $36 \%$ of severe epistaxis cases originated in the upper septum ${ }^{(7)}$, which may be consistent with our findings ( $28.3 \%$ S-point $+7.5 \%$ non-S-point upper septum $=$ $35.8 \%)$.

In addition to the high prevalence of the S-point in severe epistaxis, another relevant finding of the present study is the high prevalence of superior epistaxis, instead of posterior epistaxis. Prior studies which provided detailed descriptions of bleeding sites also presented a predominance of superior epistaxis. limura 
et al. reported that $23.4 \%$ of bleeds originated from the olfactory groove; Chiu et al. found that $36 \%$ originated in the upper septum ${ }^{(7,10)}$. The present study is novel insofar as it demonstrates that the superior portion of the nose is the leading source of severe epistaxis, rather than the posterior region. In just over half of the SEA performed in our sample, superior epistaxis was identified, while the posterior epistaxis accounted for only $26.4 \%$ of bleeding sites, thus changing the paradigm that severe epistaxis was usually of posterior origin.

Recent guidelines recommended surgery when conservative management strategies have failed, including uncontrolled epistaxis despite optimal packing, controlled epistaxis through optimal packing that recurs on attempted removal of packing, or recurrent epistaxis even if successfully treated with packing $^{(13)}$. However, all studies comparing surgery to nasal packing in severe epistaxis reported higher success rates for surgical ligation, and early vessel ligation was associated with lower cost, reduced length of stay and fewer blood transfusions, supporting early surgical intervention ${ }^{(14)}$, as we proceeded in our hospital. Current optimum surgery comprises general anesthesia with endoscopic nasal cavity examination, electrocautery of identified bleeding points, and ESPA on the affected site ${ }^{4}$. Nevertheless. our data support avoidance of ESPA when a bleeding point is identified on SEA. We advocate ESPA only if no bleeding point is found on SEA, with additional EAEA in elderly, post-trauma, rebleeding or suspected superior epistaxis cases.

\section{Conclusion}

In conclusion, systematic endoscopic assessment under general anesthesia effectively identified bleeding sites in $69.8 \%$ of cases of severe epistaxis. The S-point was the most identified bleeding site $(28.3 \%)$, followed by the lateral middle turbinate $(9.4 \%)$, non- S-point upper septum (7.5\%), nasal roof (7.5\%), and upper lateral wall (7.5\%). Finally, superior epistaxis corresponded to more than half of the identified bleeding sites, demonstrating the importance of examining this region judiciously in patients with severe epistaxis.

\section{Authorship contribution}

CNL, GRLL and VSS concepted the work, collected the data and wrote the paper. TCC, LSL and MVFM collected, analysed and interpreted the data. MST, LB and AS concepted the work and critically revised the paper. EMK concepted the work, interpreted the data and critically revised the paper.

\section{Conflict of interest}

The authors declare no conflict of interests.

\section{References}

1. Kosugi EM, Balsalobre L, Mangussi Gomes $J$, et al. Breaking paradigms in severe epistaxis: the importance of looking for the S-point. Braz J Otorhinolaryngol 2018; 84: 290-297

2. Lin G, Bleier B. Surgical Management of Severe Epistaxis. Otolaryngol Clin North Am 2016: 49: 627-637.

3. Saraceni Neto P, Nunes LMA, Gregório LC, Santos RDP, Kosugi EM. Surgical treatment of severe epistaxis: an eleven-year experience. Braz J Otorhinolaryngol 2013; 79 . 59-64.

4. Lakhani R, Syed I, Qureishi A, Bleach N. The Wexham Criteria: defining severe epistaxis to select patients requiring sphenopalatine artery ligation. Eur Arch Otorhinolaryngol 2013; 270: 2039-2043.

5. Mangussi-Gomes J, Enout MJR, Castro TC de, de Andrade JSC, Penido N de O, Kosugi EM. Is the occurrence of spontaneous epistaxis related to climatic variables? A retrospective clinical, epidemiological and meteorological study. Acta Otolaryngol 2016; 136: 1184-1189.

6. Vinay Kumar M, Raghavendra Prasad
K, Belure Gowda P, Manohar S, Chennaveerappa P. Rigid nasal endoscopy in the diagnosis and treatment of epistaxis. J Clin Diagnostic Res 2013; 7: 831-833.

7. Chiu TW, McGarry G. Prospective clinical study of bleeding sites in idiopathic adult posterior epistaxis. Otolaryngol Head Neck Surg 2007; 137: 390-393.

8. Thornton M, Mahesh B, Lang J. Posterior epistaxis: identification of common bleeding sites. Laryngoscope 2005; 115: 588-590.

9. Yukitatsu Y, Tsuzuki K, Takebayashi $H_{\text {, }}$ Sakagami M. Clinical Study of 1,515 patients presenting with epistaxis over the last 6 years. ORL 2016; 78: 232-240.

10. limura J, Hatano A, Ando Y, Arai C, Arai S, Shigeta $Y$, et al. Study of hemostasis procedures for posterior epistaxis. Auris Nasus Larynx 2016; 43: 298-303.

11. Turri-Zanoni M, Arosio AD, Stamm AC, et al. Septal branches of the anterior ethmoidal artery: anatomical considerations and clinical implications in the management of refractory epistaxis. Eur Arch Oto-RhinoLaryngology 2018; 275: 1449-1456.

12. Almeida GS, Diógenes CA, Pinheiro SD. Nasal endoscopy and the localization of the bleeding source in the epistaxis: the revolution of the last decade. Braz Otorhinolaryngol 2005; 71: 146-148.

13. Ellis M, Hall A, Hardman J, et al. The British Rhinological Society multidisciplinary consensus recommendations on the hospital management of epistaxis. J Laryngol Otol 2017; 131: 1142-1156.

14. Swords C, Patel A, Smith ME, Williams RJ Kuhn I, Hopkins C. Surgical and interventional radiological management of adult epistaxis: Systematic review. J Laryngol Otol 2017; 131: 1108-1130

Eduardo Macoto Kosugi

Rhinology Section

Federal University of Sao Paulo

Sao Paulo, SP

Brazil

Tel: $+55-11-25973340$

E-mail: edumacoto@gmail.com

This paper contains supplementary video materials online at www.rhinologyjournal.org 